\title{
Orbital involvement by NUT midline carcinoma: new presentation and encouraging outcome managed by radiotherapy combined with tyrosine kinase inhibitor: a case report
}

\author{
Peiwei Chai ${ }^{1,2+}$, Chuandi Zhou ${ }^{1,2+}$, Renbing Jia ${ }^{1,2^{*}}$ and Yefei Wang ${ }^{1,2^{*}}$
}

\begin{abstract}
Background: NUT midline carcinoma (NMC) is a poorly differentiated squamous cancer with a median survival at less than 7 months. NMC is resistant to conventional chemotherapies and characterized by rearrangement of the NUT gene.

Case presentation: Here, we described a patient who initially presented with epiphora, and an orbit involved NMC. In addition, we for the first time demonstrated that local radiotherapy combined with tyrosine kinase inhibitor (TKI) could significantly inhibit tumor progression in orbital involvement by NMC.

Conclusions: Our study for the first time described an orbit involved NMC patient initially presented with epiphora. In addition, we provided an alternative to the management of orbit involved NMC.
\end{abstract}

Keywords: NUT midline carcinoma, Epiphora, Anlotinib, Tyrosine kinase inhibitor

\section{Background}

NUT midline carcinoma (NMC) is a rare tumor arising primarily in the midline with a median survival at less than 7 months $[1,2]$. NMC is resistant to conventional chemotherapies and characterized by rearrangement of the NUT gene [3]. To our best knowledge, no report has documented an NMC with primary presentation of epiphora. Due to the rapid progression of NMC, the patient missed the best chance of surgical resection. Notably, the recent availability of targeted therapy with Bromo- and Extra-Terminal domain inhibitors (BETi) may hold promise in treating NMC. However, BETi is not currently available in China. In this report, we performed local radiotherapy with Anlotinib, which significantly inhibited NMC progression and could provide an example of an alternative therapeutic option for NMC.

\footnotetext{
* Correspondence: renbingjia@sjtu.edu.cn; paper34@163.com

†Peiwei Chai and Chuandi Zhou contributed equally to this work.

${ }^{1}$ Department of Ophthalmology, Ninth People's Hospital, Shanghai JiaoTong University School of Medicine, Shanghai 200025, People's Republic of China Full list of author information is available at the end of the article
}

\section{Case presentation}

A 59-year-old female presented with 14 days of epiphora. She denied vision loss, pain, epistaxis, or fevers. Her complete blood count examination showed $\mathrm{Hb} 112 \mathrm{~g} / \mathrm{L}$ [ref. $113 \sim 151 \mathrm{~g} / \mathrm{L}$ ], WBCs $4.2 \times 10^{9} / \mathrm{L}$ [ref. $4.0 \sim 10.0 \times$ $10^{9} / \mathrm{L}$ ], and Platelets $213 \times 10^{9} / \mathrm{L}$ [ref. $100 \sim 300 \times 10^{9} / \mathrm{L}$ ]. Her renal function test (RFL), liver function test (LFT) and lipid profile were within normal limits (DBIL $2.0 \mu \mathrm{mol} /$ $\mathrm{L} \quad[\mathrm{ref} . \quad 0.51 \sim 3.42 \mathrm{umol} / \mathrm{L}], \quad \mathrm{I}-\mathrm{BIL} \quad 2.4 \mu \mathrm{mol} / \mathrm{L} \quad[\mathrm{ref}$. 1.71 13.8umol/L], Scr $66 \mu \mathrm{mol} / \mathrm{L}$ [ref. 30 110umol/L], BUN $3 \mathrm{mmol} / \mathrm{L}$ [ref. $2.9 \sim 7.5 \mathrm{mmol} / \mathrm{L}$ ] and LDL $2.78 \mathrm{mmol} /$ $\mathrm{L}$ [ref. 1.9 3.6 mmol/L]). Irrigation of the lacrimal passage suggested no blockage, no purulent or hemorrhagic discharge. Three months later, the symptom of epiphora aggravated. Orbital computed tomography (CT) and magnetic resonance imaging (MRI) scans showed a right orbital mass extending to the adjacent paranasal sinuses (Fig. 1). The results of a gastrointestinal tract endoscopy, colonoscopy, nasal endoscope and ${ }^{18} \mathrm{~F}$-2-Fluoro-2-Deoxy-D-Glucopyranose positron emission tomography $\left({ }^{18} \mathrm{~F}\right.$-FDG PET/ 


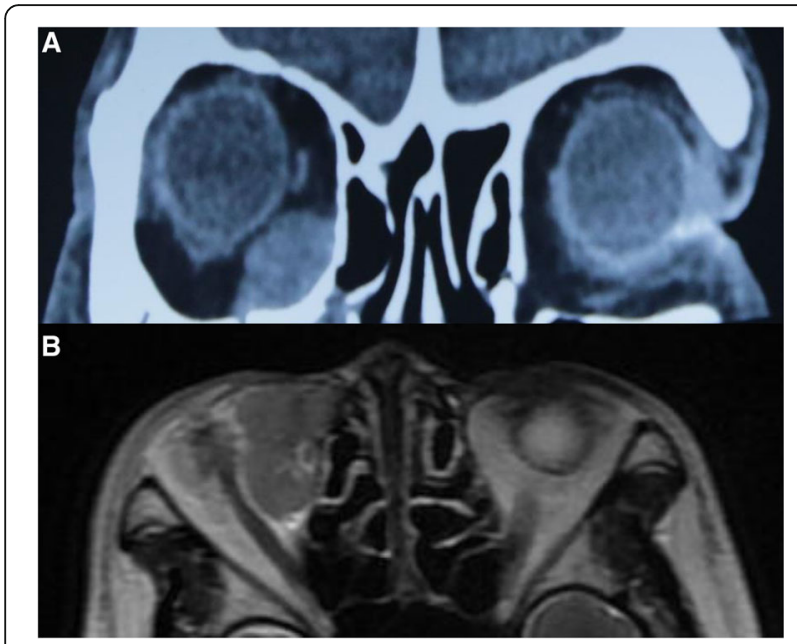

Fig. 1 An orbit involved NUT midline carcinoma (NMC). a The orbital computed tomography $(\mathrm{CT})$ and (b) Magnetic resonance imaging (MRI) showed an orbital mass extending to the adjacent paranasal sinuses
CT) revealed no other malignancies (Fig. 2). The mass was surgically removed. Pathologic analysis suggested a malignant epithelial neoplasm with squamous features with direct juxtaposition of basaloid, immature and undifferentiated squamous cells (Fig. 3). A panel of immunohistochemistry stains showed positive staining for markers of squamous differentiation, for p40(+), p63(+), CK5/6(+), NUT (+) and Ki67(50\%+). Fluorescent DNA in situ hybridization (FISH) demonstrated the presence of NUT-BRD4 rearrangement (Fig. 4). In this condition, orbital exenteration was indicated, however, the patient refused. The mass grew rapidly after primary resection, which metastasized to cervical lymph node 2 months later (pathologically proved with biopsy). The patient developed severe dyspnea and could hardly perform prostrations (Fig. 5, 8-month). 4 months later, the patient was treated with first round of local radiotherapy (50 Gy/25 Fx), tumors shrunk, and the symptom of dyspnea eased. No remarkable adverse effects were observed. However, 2 months later, more metastasis was observed in the forehead and neck (Fig. 5, red arrow). The patient was then treated with multi-targeting tyrosine kinase inhibitor (Anlotinib, $12 \mathrm{mg}$, qd) and second round of local radiotherapy (50 Gy/25 Fx) thereafter. The masses continued to shrink,

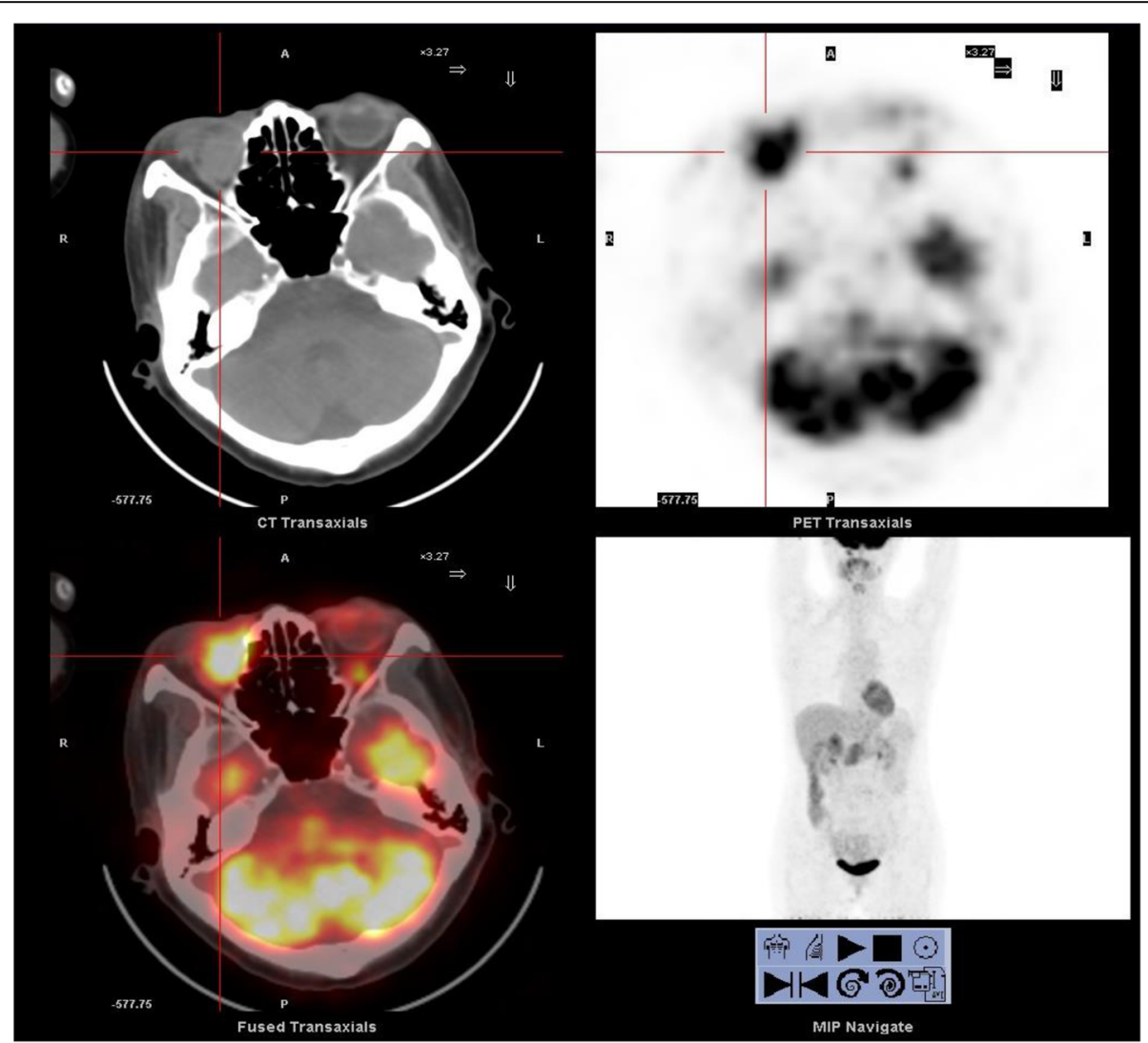

Fig. 2 A systemic ${ }^{18} \mathrm{~F}$-2-Fluoro-2-Deoxy-D-Glucopyranose positron emission tomography $\left({ }^{18} \mathrm{~F}-\mathrm{FDG} \mathrm{PET} / \mathrm{CT}\right)$ demonstrated an orbital mass with increased FDG uptake but no other remarkable malignancy in the trunk 


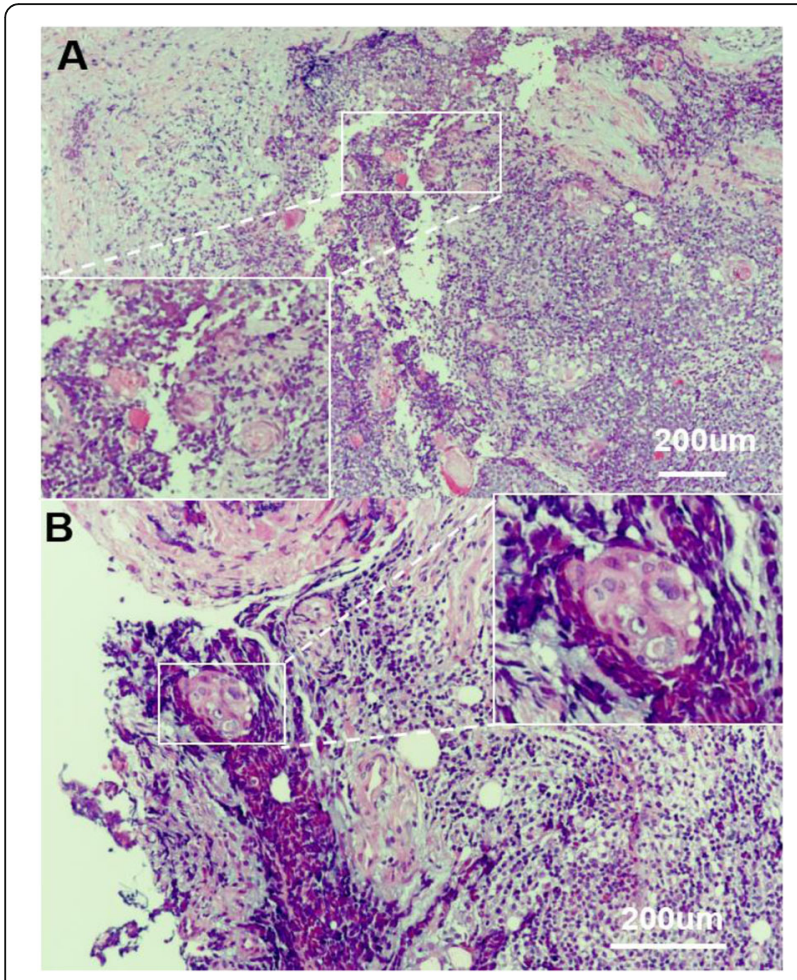

Fig. 3 Hematoxylin-eosin (HE) staining demonstrated a NUT midline carcinoma with aberrant squamous differentiation. $\mathbf{a}-\mathbf{b}$ : A malignant epithelial neoplasm with squamous features with direct juxtaposition of basaloid, immature and undifferentiated squamous cells. Scale bar: 50um and the lymph node metastasis was significantly decreased (Fig. 5, 18-month). Except for gingival bleeding, no other serious adverse effects have been observed. To date, the patient had an 8-month disease-free survival.

\section{Discussion and conclusions}

NMC is an aggressive subtype of squamous cell carcinoma, which is caused by chromosomal rearrangement of the gene nuclear protein in testis (NUT) on $15 q 14$ [4,5]. In $80 \%$ of NMC patients, the chromosomal translocation occurs between the NUT and BRD4 genes, leading to the occurrence of a BRD4-NUT fusion gene [6]. To date, a standard treatment for NMC has not been established and a multitarget treatment strategy with systemic chemotherapy, surgery and radiation therapy is currently adopted in clinical practice [7]. For this patient, BRD4-NUT encoded fusion proteins, which block epithelial cell differentiation and contribute to carcinogenesis, were examined by FISH assay. Although NMC remains an underrecognized squamous carcinoma, there has appeared to be increasing awareness of disease and frequency of diagnosis [8]. Typically, NMC arises from midline supradiaphragmatic structures: the upper aerodigestive tract (50\%) and the mediastinum (41\%). Moreover, rarer cases have been observed below the diaphragm (bladder) and outside the midline axis (major salivary glands, iliac bone, adrenal gland, and pancreas). Here, we for the first time revealed that the NMC, initially presented with epiphora.

Previous studies have presented grave prognoses for NMC, with a limited life expectancy and a $>80 \%$ chance of death within the first year of diagnosis [9]. Additionally,

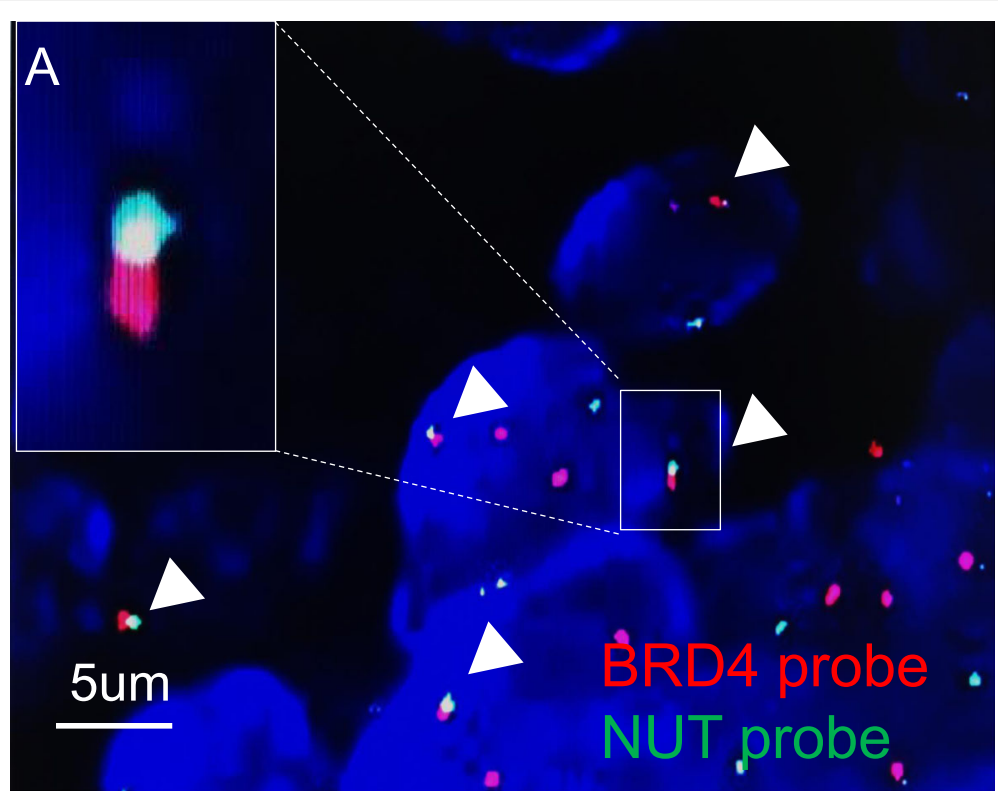

Fig. 4 Fluorescent DNA in situ hybridization (FISH) demonstrated a BRD4-NUT rearrangement (White triangle). A red probe that spans NUT splits and joins the green BRD4 centromeric probe. Scale bar: 5 um 


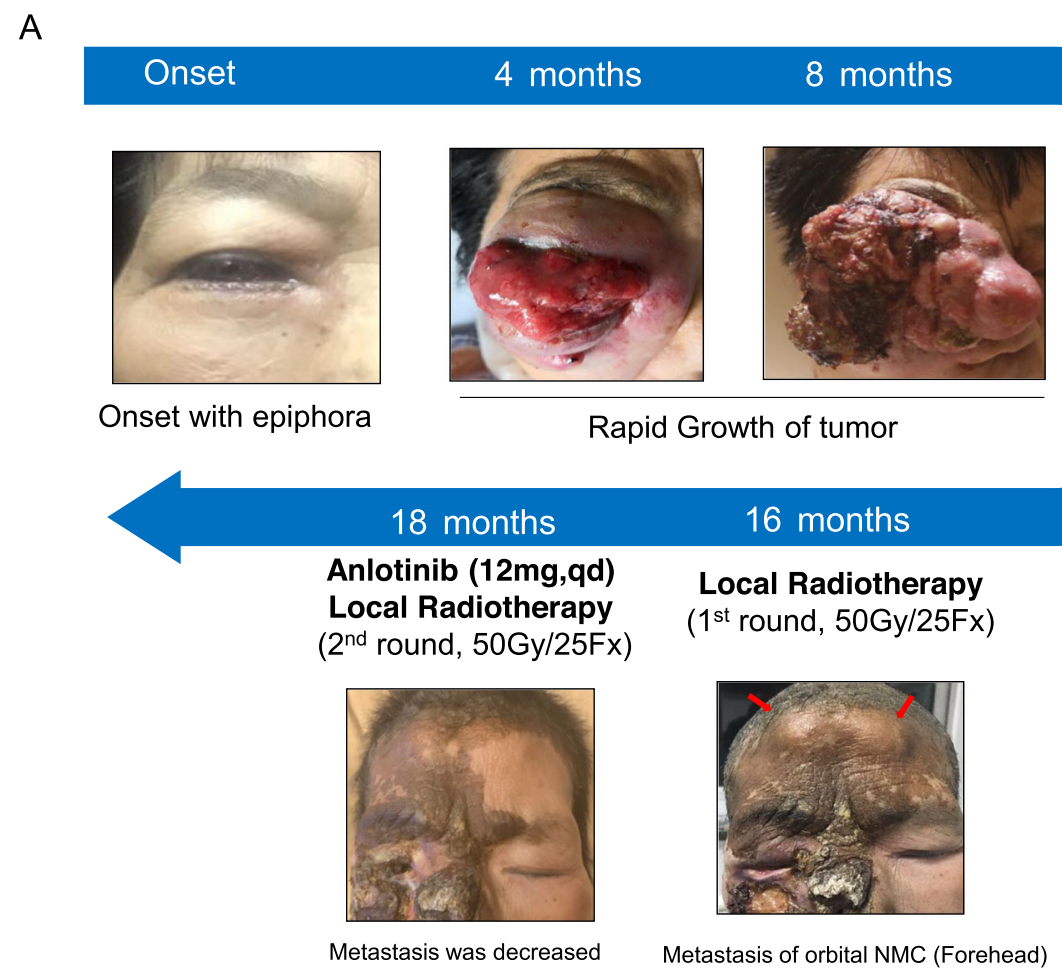

Fig. 5 The appearance of the NUT midline carcinoma (NMC) patient at the time of onset, 4, 8, 16 and 18 months after diagnosis. The patient's consent for using their photos for publication has been obtained

aggressive surgical resection and early radiotherapy may prolong life expectancy for the non-metastatic NMC patient [4]. In this case, we assumed that the NMC grew rapidly after primary resection was due to the incomplete resection. And the patient refused orbital exenteration for disfigurement of facial appearance. The recent availability of targeted therapy with acetyl-histone mimics (BETi) may hold promise in treating NMC [2]. Jeremy Lewin et al. found that NMC had a partial response with 1.4 to 8.4 months of BETi treatment [10]. However, BETi is not currently available in China. In this report, we performed local radiotherapy combined with multitargeting tyrosine kinase inhibitor (Anlotinib) could significantly suppress NMC progression, which might provide an alternative to the treatment of NMC.

Receptor tyrosine kinases (RTKs) are transmembrane glycoproteins that communicate with cellular growth factors and extracellular ligands [11]. They play vital roles in intracellular tyrosine phosphorylation and intracellular signaling [12]. RTK activation mediates many vital physiological processes, including proliferation, migration, differentiation and apoptosis [13]. In addition, RTKs have been implicated in a variety of cancers, such as non-small-cell lung cancer (NSCLC), renal cell carcinoma (RCC) and advanced medullary thyroid cancer [13]. Anlotinib is a novel, orally administered RTKs inhibitor that multi-targets fibroblast growth factor receptor (FGFR), vascular endothelial growth factor receptor (VEGFR) and platelet-derived growth factor receptors (PDGFR) [14]. Compared to placebo, it largely improved both progression-free survival (PFS) and overall survival (OS) in a phase III trial in patients with advanced nonsmall-cell lung cancer (NSCLC) [15]. Here, we for the first time demonstrated that the clinical application of Anlotinib in treating with orbital NMC.

In summary, we initially reported a case of NMC with the primary complaint of epiphora. In addition to routine tests of lacrimal duct irrigation, a CT or MRI should be carried out if the symptoms persist. Heightened awareness and early recognition of NMC are critical, and the clinician should possess adequate suspicion for NMC, especially when encountering patients with refractory epiphora. NMC is histopathologically characterized by abrupt squamous differentiation, a NUT immunostaining positive result and BRD4-NUT rearrangement by FISH assay. Once diagnosed with NMC, complete surgical resection should be performed. Adequate radiotherapy is recommended postoperatively. Additionally, other adjuvant therapies, such as BETi, local radiotherapy and tyrosine kinase inhibitor, could also be considered for NMC treatment. Larger studies and longer follow up time still needed to validate our finding. 


\section{Abbreviations}

BETi: Bromo- and Extra-Terminal domain inhibitors; CT: Computed tomography; FGFR: Fibroblast growth factor receptor; FISH: Fluorescent DNA in situ hybridization; LFT: Liver function test; MRI: Magnetic resonance imaging; NMC: NUT midline carcinoma; NUT: Nuclear protein in testis; PDGFR: Platelet-derived growth factor receptors; PET: Positron emission tomography; RFL: Renal function test; RTKs: Receptor tyrosine kinases; VEGFR: Vascular endothelial growth factor receptor

\section{Acknowledgements}

We thank the patient for granting permission to publish this information and wish her a good health.

\section{Authors' contributions}

Conception and design: PC, RJ; Financial support: CZ, RJ; Administrative support: XF; Provision of study materials or patients: PC; Collection and assembly of data: CZ; Data analysis and interpretation: PC; Manuscript writing: All authors; Final approval of manuscript: All authors; Accountable for all aspects of the work: All authors. All authors have read and approved the manuscript.

\section{Funding}

The National Natural Science Foundation of China (grants 81570884 81770961), the SMC-ChenXing Yong Scholar Program (2016, Class A) and the Shanghai Municipal Education Commission-Gaofeng Clinical Medicine Grant (20152223). Innovation Fund for Translational Medicine (15ZH1005), Ph.D. Programs Foundation of Shanghai JiaoTong University School of Medicine (BXJ201834), Innovative research team of high-level local universities in Shanghai (Department of Ophthalmology, Shanghai Ninth People Hospital). The Interdisciplinary Program of Shanghai Jiao Tong University (YG2016MS02). The funders had no role in the design of the study nor in the collection, analysis and interpretation of data or in writing the manuscript.

\section{Availability of data and materials}

N.A

\section{Ethics approval and consent to participate}

Ethics approval for this study was obtained from the Shanghai Jiaotong University. Only non-identifiable information is presented. The patient provided signed informed consent for an Institutional Review Board-approved protocol for research use of medical records, of pathologic specimens obtained as part of routine clinical care, and publication.

\section{Consent for publication}

Written informed consent to publish the clinical data was obtained from the patient before the initiation of the study.

\section{Competing interests}

The authors declare that they have no competing interests.

\section{Author details}

'Department of Ophthalmology, Ninth People's Hospital, Shanghai JiaoTong University School of Medicine, Shanghai 200025, People's Republic of China. ${ }^{2}$ Shanghai Key Laboratory of Orbital Diseases and Ocular Oncology, Shanghai, China.

Received: 6 November 2019 Accepted: 23 December 2019

Published online: 04 January 2020

\section{References}

1. Chau NG, Hurwitz S, Mitchell CM, et al. Intensive treatment and survival outcomes in NUT midline carcinoma of the head and neck. Cancer. 2016; 122:3632-40.

2. French C. NUT midline carcinoma. Nat Rev Cancer. 2014;14:149-50.

3. Beesley AH, Stirnweiss A, Ferrari E, et al. Comparative drug screening in NUT midline carcinoma. Br J Cancer. 2014:110:1189-98.

4. Bauer DE, Mitchell CM, Strait KM, et al. Clinicopathologic features and longterm outcomes of NUT midline carcinoma. Clin Cancer Res. 2012:18:5773-9.

5. Wang R, Cao XJ, Kulej K, et al. Uncovering BRD4 hyperphosphorylation associated with cellular transformation in NUT midline carcinoma. Proc Natl Acad Sci U S A. 2017;114:E5352-61.
6. D'Souza JN, Notz G, Bogdasarian RN, et al. Orbital involvement by NUT midline carcinoma. Ophthalmic Plast Reconstr Surg. 2015;31:e147-50.

7. Bragelmann J, Dammert MA, Dietlein F, et al. Systematic kinase inhibitor profiling identifies CDK9 as a synthetic lethal target in NUT midline carcinoma. Cell Rep. 2017;20:2833-45.

8. Gokmen-Polar Y, Kesler K, Loehrer PJ Sr, et al. NUT midline carcinoma masquerading as a Thymic carcinoma. J Clin Oncol. 2016;34:e126-9.

9. Teo M, Crotty P, O'Sullivan M, et al. NUT midline carcinoma in a young woman. J Clin Oncol. 2011:29:e336-9.

10. Lewin J, Soria JC, Stathis A, et al. Phase Ib trial with Birabresib, a smallmolecule inhibitor of Bromodomain and Extraterminal proteins, in patients with selected advanced solid tumors. J Clin Oncol. 2018;36:3007-14.

11. Revach OY, Sandler O, Samuels Y, et al. Cross-talk between receptor tyrosine kinases AXL and ERBB3 regulates Invadopodia formation in melanoma cells. Cancer Res. 2019:79:2634-48.

12. Leopold AV, Chernov KG, Shemetov AA, et al. Neurotrophin receptor tyrosine kinases regulated with near-infrared light. Nat Commun. 2019;10:1129.

13. Wahl DR, Lawrence TS. No sugar added: a new strategy to inhibit Glioblastoma receptor tyrosine kinases. Clin Cancer Res. 2019;25:455-6.

14. Chen XZ. Anlotinib for refractory advanced non-small cell lung Cancer in China. JAMA Oncol. 2019;5:116-7.

15. Han B, Li K, Wang Q, et al. Effect of Anlotinib as a third-line or further treatment on overall survival of patients with advanced non-small cell lung Cancer: the ALTER 0303 phase 3 randomized clinical trial. JAMA Oncol. 2018;4:1569-75

\section{Publisher's Note}

Springer Nature remains neutral with regard to jurisdictional claims in published maps and institutional affiliations.
Ready to submit your research? Choose BMC and benefit from:

- fast, convenient online submission

- thorough peer review by experienced researchers in your field

- rapid publication on acceptance

- support for research data, including large and complex data types

- gold Open Access which fosters wider collaboration and increased citations

- maximum visibility for your research: over $100 \mathrm{M}$ website views per year

At $\mathrm{BMC}$, research is always in progress.

Learn more biomedcentral.com/submissions 